\title{
A NEW MULTIMODAL MULTI-CRITERIA ROUTE PLANNING MODEL BY INTEGRATING A FUZZY-AHP WEIGHTING METHOD AND A SIMULATED ANNEALING ALGORITHM
}

\author{
Fazel Ghaderi ${ }^{a}$, Parham Pahlavani ${ }^{b^{*}}$ \\ ${ }^{\text {a }}$ MSc. Student in GIS Division, School of Surveying and Geospatial Eng., College of Eng., University of Tehran, Tehran, Iran \\ - fazel.ghaderi@ut.ac.ir \\ ${ }^{\mathrm{b}}$ Assistant Professor, Center of Excellence in Geomatics Eng. in Disaster Management, School of Surveying and Geospatial Eng., \\ College of Eng., University of Tehran, Tehran, Iran- Pahlavani@ut.ac.ir
}

KEY WORDS: Route planning, Multi-criteria, Fuzzy-AHP, Simulated annealing

\begin{abstract}
:
A multimodal multi-criteria route planning (MMRP) system provides an optimal multimodal route from an origin point to a destination point considering two or more criteria in a way this route can be a combination of public and private transportation modes. In this paper, the simulate annealing (SA) and the fuzzy analytical hierarchy process (fuzzy AHP) were combined in order to find this route. In this regard, firstly, the effective criteria that are significant for users in their trip were determined. Then the weight of each criterion was calculated using the fuzzy AHP weighting method. The most important characteristic of this weighting method is the use of fuzzy numbers that aids the users to consider their uncertainty in pairwise comparison of criteria. After determining the criteria weights, the proposed SA algorithm were used for determining an optimal route from an origin to a destination. One of the most important problems in a meta-heuristic algorithm is trapping in local minima. In this study, five transportation modes, including subway, bus rapid transit (BRT), taxi, walking, and bus were considered for moving between nodes. Also, the fare, the time, the user's bother, and the length of the path were considered as effective criteria for solving the problem. The proposed model was implemented in an area in centre of Tehran in a GUI MATLAB programming language. The results showed a high efficiency and speed of the proposed algorithm that support our analyses.
\end{abstract}

\section{INTRODUCTION}

One of the most important problem of the modern societies is the way to encourage people to use public transportation modes (i.e. bus, subway) instead of their private car. Increase in use of public transportation modes leads to have better traffic flow and to reduce the congestion and the $\mathrm{CO}_{2}$ emission. A journey in a metropolis usually is a combination of public, i.e. bus and subway and private, i.e. private car and walking, transportation modes. People for travelling within a metropolis prefer to cross from a route that is an appropriate combination of transportation modes that satisfy their criteria. Todays, many online services, e.g. Google Transit, help people in their journeys. The most problem of such services is the lack of ability of properly combination of the public and private transportation modes in addition to ignore of considering the importance of each criterion according to user preferences in route planning process. The main purpose of this research is to provide a MMRP system that provides the best multimodal route to users according to their assigned preferences to each criterion. Because of importance of route planning in metropolises, many researches have studied in this field are divided into three major categories as follows:

- Multi-modal route planning: most of these researches were related to find a shortest multimodal route in a static transportation network [(Crainic \& Rousseau, 1986); (Nguyen, Morello, \& Pallottino, 1988); (Delavar, Samadzadegan, \& Pahlavani, 2004)] although some researches implemented dynamic ones (Davies \& Lingras, 2003). (Yu \& Lu, 2012) used genetic algorithm to find multimodal shortest route in a static transportation network. They defined multimodal routes as chromosomes with variable length with several parts in a way each part presented a type of transportation. (Abbaspour \& Samadzadegan, 2011) used an adapted evolutionary algorithm with variable length chromosomes to find shortest multi-modal route in a complex and large dynamic transportation network. (Borole, Rout, Goel, Vedagiri, \& Mathew, 2013) used real-time transportation network data for finding shortest multimodal route. For real-time positioning of the vehicles, they used GPS.

- Multi-criteria route planning: (Huang, Cheu, \& Liew, 2004) proposed a novel method for evaluating the risk of trucks carrying hazardous materials by integrating geographical information systems (GISs) and genetic algorithm. (Pahlavani, Samadzadegan, \& Delavar, 2006) succeed to improve the rate of searches in urban multi-criteria route planning in a real transportation network with unspecified site satisfaction by considering multi dependent criteria. In this research, they integrated GIS and GA utilization. (Liu, Mu, Luo, \& Li, 2012) used an oriented spanning tree based on simulated annealing (SA) for solving shortest path problem in a single mode transportation network. The most important reason of using oriented spanning tree in their research was improving in local and global search of the designed SA algorithm. (Pahlavani, Delavar, \& Frank, 2012) proposed a modified invasive weed optimization algorithm to solve the personalized urban multicriteria quasi-optimum path problem. In comparison to the GA algorithm, their proposed algorithm had better characteristics, such as better quality metric and lower running time. Also, (Pahlavani \& Delavar, 2014) integrated fuzzy algorithms and artificial neural networks (ANNs) for modeling driver preferences.

- Multi-modal multi-criteria researches: (Abdelghany \& Mahmassani, 2001) proposed a new algorithm for finding the 
best multimodal route in a transportation network. Their proposed algorithm was based on a non-dominated route planning algorithm that determined a set of non-dominated routes. Finally, the best route was calculated among these routes, based on the general cost function. (Qu \& Chen, 2008) integrated a fuzzy AHP weighting method with an ANN for finding optimal multimodal route in a static transportation network. They used AHP method for calculating initial criteria weights to improve the efficiency of the ANN. (Antsfeld \& Walsh, 2012) proposed a new algorithm for finding the best route in a multimodal public transportation network. Their proposed a model by considering many realistic features, such as walking between transport modes stations, multiple objectives, traffic days, and finding connections between geographical locations rather than only origin and destination points. (Bouhana, Fekih, Abed, \& Chabchoub, 2013) proposed a novel algorithm by integrating a case-based reasoning and Choquet integral in order to determine optimal route in a transportation network. The main capability of their proposed algorithm was predicting users' preferences for give a context to help them with facing a new situation. (Liu, Yang, Mu, Li, \& $\mathrm{Wu}, 2014)$ used a label correcting approach for finding the best multimodal multi-criteria route by considering transfer delaying and arriving time window.

In this research, the fuzzy AHP weighting method is used for helping users to determine the importance of each criterion In this method, a matrix is provided for comparison between importance of a pair of criteria rather than others that this importance must be assigned with fuzzy numbers. Using fuzzy numbers provides users to consider their uncertainty in a pairwise comparison between criteria. In this research, the triangular fuzzy numbers are considered for pairwise comparisons. After determining the effective criteria, their weight values of each edge for all criteria must be normalized. In next step, an algorithm has been proposed for finding the optimal multimodal route. MMRP is a NP-hard problem. For a NP-hard problem it is generally believed that no algorithm exists to solve each instance in polynomial time. One of the methods for solving this type of problems is using heuristic algorithms like SA. One of the most important problems in a heuristic algorithm is trapping in local minima. For solving this problem, SA algorithm imposes different randomized search, acceptance, and stopping criteria on the local search method. In this research, because a trip in the determined case study is no longer than three hours, Tehran transport network data between 5:30 AM to 8:30 AM of a day were used.

The reminder of this paper consists of 6 sections. Problem definition is illustrated in Section 2. The proposed method for calculating criteria weights is presented in Section 3. Criteria modelling are illustrated in Section 4. In Section 5, the proposed SA algorithm is shown. Experimental results are discussed in Section 6, and finally conclusions and future works are presented in Section 7.

\section{PROBLEM DEFINATION}

The main purpose of MMRP is providing an optimal multimodal route between origin and destination points by considering the weights of the effective criteria. A transportation network can be represent as a directed graph $G=(V, E)$, in which $V$ and $E$ represents nodes and edge sets, respectively. Connectivity is an important aspect of a transportation network that a route planning system would be implemented on it. In this research, five transportation modes, including taxi, subway, bus rapid transit (BRT), subway, and walking are considered for traveling between nodes. In this research, these five sub-graphs were connected with walking edges. Flow chart of the proposed algorithm for route planning is shown in Fig 1.

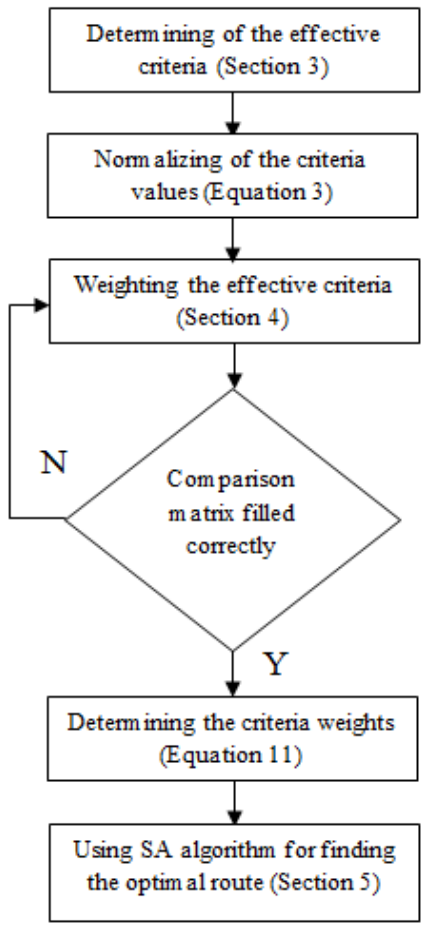

Figure 1. The flow chart of the proposed model

In the first step, all criteria that might be important for a passenger in using public transportation system must be determined. Next, the criteria values must be normalized. In the third step, a pairwise comparison between criteria must be applied by the fuzzy numbers. After filling this pairwise comparison matrix, the consistency of the assigned numbers in pairwise comparison matrix must be evaluated. After proving that the comparison matrix has been filled correctly, the criteria weights will be calculated. Finally, the optimal route will be determined with the SA algorithm.

\section{CRITERIA MODELING}

An important and critical problem in route planning problem is the formulation of the effective criteria. Criteria modelling not only consists of how to calculate each criterion value, but also consists of how to normalize their value. In this research, four independent criteria, including time, length, fare, and users' bother across route as follows: (I) Length: after determining this criterion value for each link, their normalized value were calculated according to Eq 3. (II) Time: for BRT, subway, and bus links their values were calculated according to their timetable. The link values of walking and taxi modes were calculated according to average speed of them. Their normalized value were calculated according to Eq 3. (III) Fare: The value of this criterion for walking links are zeros. Each link of bus and BRT modes has its specific value. For subway and taxi modes, these values depend on the length of the route and their normalized value would be calculated based on Eq 3. (IV) Users' bother: users often like to use specific transportation modes. For instance, some passengers prefer to use taxi and the others prefer to use BRT. Users' preferences in traveling with different transportation modes is shows in Table 1. 
Table 1. Guidelines for determining users' bother

\begin{tabular}{cc}
\hline Mode & Bother \\
\hline taxi & 1 \\
bus & 2 \\
BRT & 3 \\
Subway & 4 \\
walking & 5 \\
\hline
\end{tabular}

This criterion value calculates as follow: Let $n$ be the number of segments of the route. Let $l_{p}$ and $b_{p}$ be the length and users' bother across the $p^{\text {th }}$ segment of the route $(p=1$ to $n)$, then the weight of this segment will be calculated as follow:

$$
w_{p}=\frac{l_{p}}{\sum_{p=1}^{n} l_{p}} \text {. }
$$

The final value of this criterion for this route will be calculated as follow:

$$
u b=\sum_{p=1}^{n} w_{p} \times b_{p}
$$

In a multi criteria decision making, for comparing different alternatives, the criteria values must be normalized (Nadi \& Delavar, 2011). In this research, maximum score method is used for this purpose as follow (Malczewski, 1999):

$$
c_{i j}^{\prime}=\frac{c_{i j}}{c_{\max }},
$$

where $c_{i j}^{\prime}, c_{i j}$, and $c_{\max }$ is the normalized value of the $j^{t h}$ criterion for route $i$, its original value, and the maximum value of criterion $i$ between all alternatives (routes), respectively.

\section{FUZZY ANALYTICAL HIERARCHY PROCESS METHOD}

Fuzzy analytical hierarchy process (fuzzy AHP) weighting method by Saaty (Saaty, 1980) embeds fuzzy theory to basic AHP method. In AHP weighting method, a matrix-liked structure is considered for pairwise comparison between criteria with exact numbers. Despite the general popularity, the AHP method is not able to consider the users' ambiguity and the lack of clarity in their preferences (Deng, 1999). To solve this problem of the AHP method, (Buckley, 1985) proposed to use fuzzy numbers rather than exact ones in pairwise comparisons and introduced the fuzzy AHP method. In this research, triangular fuzzy numbers which first defined by Laarhoven and Pedrycz was used for the pairwise comparisons. By considering this type of fuzzy numbers, the preference of the criterion $i$ against criterion $j$ and its converse value is indicated as follow:

$$
\begin{aligned}
& \tilde{a}_{i j}=\left(a_{i j}, b_{i j}, c_{i j}\right), \\
& \tilde{a}_{j i}=\left(1 / c_{i j}, 1 / b_{i j}, 1 / a_{j i}\right),
\end{aligned}
$$

Each triangular fuzzy number has a linear representation (Figure 2) according to its membership function (Eq. 6).

$$
\mu(z)=\left\{\begin{array}{lc}
0 & \mathrm{z}<\mathrm{a} \text { or } \mathrm{z}>\mathrm{c} \\
\frac{\mathrm{z}-\mathrm{a}}{\mathrm{b}-\mathrm{a}} & \mathrm{a} \leq \mathrm{z} \leq \mathrm{b} \\
\frac{-\mathrm{z}+\mathrm{c}}{\mathrm{c}-\mathrm{b}} & \mathrm{b} \leq \mathrm{z} \leq \mathrm{c}
\end{array}\right.
$$

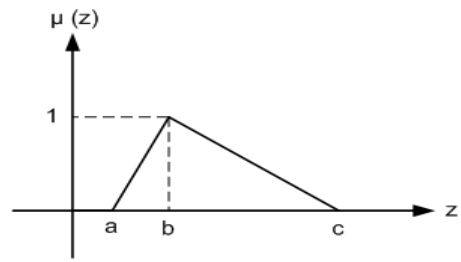

Figure 2. A triangular fuzzy number representation

The pairwise compression will be:

$A=\left[\begin{array}{ccc}\left(a_{11}, b_{11}, c_{11}\right) & \cdots & \left(a_{1 n}, b_{1 n}, c_{1 n}\right) \\ \vdots & \ddots & \vdots \\ \left(a_{n 1}, b_{n 1}, c_{n 1}\right) & \cdots & \left(a_{n n}, b_{n n}, c_{n n}\right)\end{array}\right]$

Fuzzy AHP standard scale for the pairwise comparisons was presented in Table 2.

Table 2. Fuzzy AHP preference scale to form the pairwise comparison (Buckley, 1985)

\begin{tabular}{ccc}
\hline $\begin{array}{c}\text { Fuzzy AHP scale of } \\
\text { importance for } \\
\text { pairwise comparison }\end{array}$ & Numeric rating & Reciprocal \\
\hline Absolute & $(7,9,11)$ & $(1 / 11,1 / 9,1 / 7)$ \\
Very, very strong & $(5,7,9)$ & $(1 / 9,1 / 7,1 / 5)$ \\
Very strong & $(3,5,7)$ & $(1 / 7,1 / 5,1 / 3)$ \\
Moderate & $(1,3,5)$ & $(1 / 5,1 / 3,1)$ \\
weak & $(1,2,4)$ & $(1 / 4,1 / 2,3 / 1)$ \\
Equal & $(1,1,1)$ & $(1,1,1)$ \\
\hline
\end{tabular}

In next step, fuzzy numbers must be normalized. In this research geometric mean method has been used for normalizing the fuzzy numbers (Eqs. 8 to 10) as follows:

$a_{i}=\left(\prod_{j=1}^{n} a_{i j}\right)$,

$a=\sum_{i=1}^{n} a_{i}$

Similarly, $b_{i}, c_{i}, b$, and $c$ values should be calculated. After determining these six values, the normalized value of the weight of criterion $i$ is calculated according to Eq. 10:

$\mu_{i}(z)=\left(\frac{a_{i}}{c}, \frac{b_{i}}{b}, \frac{c_{i}}{a}\right)$.

Finally, this fuzzy number must be altered by an exact number. In this research, the Centroid defuzzification method for altering this fuzzy number with an exact number was used as follow:

$Z_{i}=\frac{\int\left(\mu_{i}(z) \cdot z\right) d z}{\int \mu_{i}(z) d z}$.

An important and critical problem of the fuzzy AHP method is checking its consistency that has a very computation demands 
that is beyond of the scope of this paper. For more studies read (Leung \& Cao, 2000)).

\section{SIMULATED ANNEALING (SA) algorithm}

Simulated annealing (SA) is one of the most commonly used meta-heuristics, and has been successfully applied to solve several types of NP-Hard problems like MMRP. This algorithm first proposed for optimization by (Kirkpatrick, Gelatt, \& Vecchi, 1983). For an NP-hard problem, it is generally believed that no algorithm could solve each instance in polynomial time. One of the methods for solving this type of problems is using a meta-heuristic algorithm like SA. One of the most important problems in a meta-heuristic algorithm is trapping in local minima. This algorithm is based on the analogy with the behavior of physical annealing process in solids (Yang, Peters, $\& \mathrm{Tu}, 2005)$. The main advantage of the proposed SA algorithm is its capability of escaping from local optimum point to optimum one. The proposed SA algorithm starts from one initial answer, finds a neighbour for the answer, and if the fitness function improves, moves to that answer. It also may moves to neighbour answer even when neighbour answer would not have any improvement in the fitness function. This is due to a probability function that its value decreased with increasing the number of algorithm repetition and can be calculated as follows:

$p=e^{\left(\frac{-\Delta E}{K_{b} T}\right)}$,

where $p$ is called acceptance probability, $T$ is temperature and has bigger value in the beginning and its value reduced gradually as follows:

$T_{a+1}=\frac{T_{a}}{1+\beta \times \sqrt{T_{a}}}, \quad a=1,2, \ldots, N-1$,

where $T_{a}$ is the temperature in iteration $a, T_{a+1}$ is the temperature in in iteration $a+1$, and $N$ is number of iteration. As illustrated in Eq. 13, value of temperature decreased with model reputation. This is a critical point in SA algorithm that causes probability of accepting inferior solutions decreased with model repetition and helps to algorithm stability. Another parameter in above equation is $\beta \in(0,1)$, called cooling rate and give a control on cooling speed (Liu et al., 2012) and also its value must be tuned.

Another parameter in Eq. 12 is $K_{b}$ which is Boltzman constant. Larger value for $K_{b}$ increases the probability of accepting inferior solutions and by considering the large value, the stability of the solutions may be lost. Also, this parameter must be tuned. Next parameter in this equation is $\Delta E$ that is calculated by

$\Delta E=E(x)-E\left(x^{\prime}\right)$,

where $x$ and $x^{\prime}$ are a solution and its neighbour respectively and $E(x)$ and $E\left(x^{\prime}\right)$ are their fitness values. Fitness value for a solution (route) in this paper is calculated as follows:

$E(r)=\sum_{i=1}^{4} w_{i} \times x_{i r}$,

where $w_{i}$ is the weight of criterion $i$ and $x_{i r}$ is the value of criterion $i$ for route $r$. Figure 3 shows the flowchart of the proposed SA algorithm.

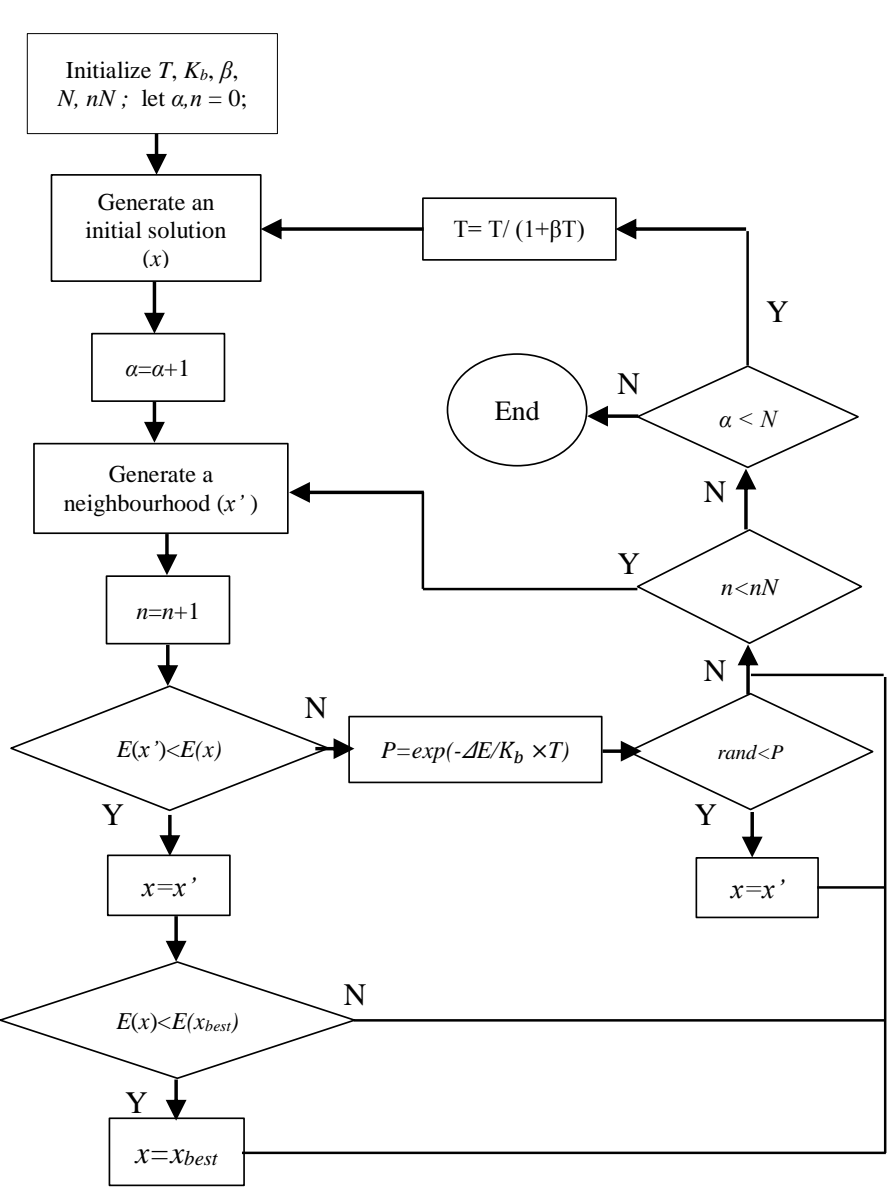

Figure 3. The proposed SA algorithm

\subsection{Generating neighborhood solutions}

Suppose $x$ as a solution. This route is a set of nodes and arcs $(x=\{1,(1,2), 2,(2,3), \ldots, 9,(9,10)\})$. Also we can present this route as a vector of its nodes (Figure 4 ).

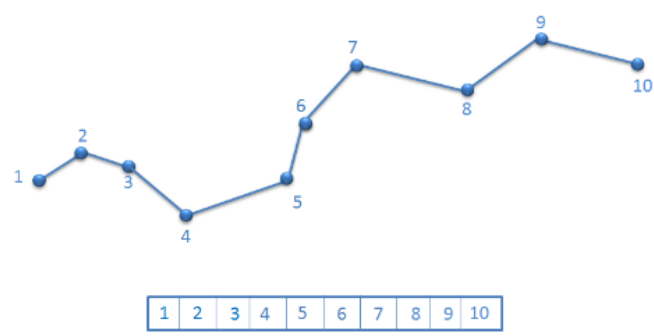

Figure 4. An instance solution and its representation as a vector

For generating a neighbourhood solution for this solution, at first, two nodes of this route must be selected randomly, e.g., for example node 3 and node 8 . Then a new route must be generated between these two nodes. Finally, neighbourhood solution is obtained by integrating the parts as illustrated in Figure 5. 


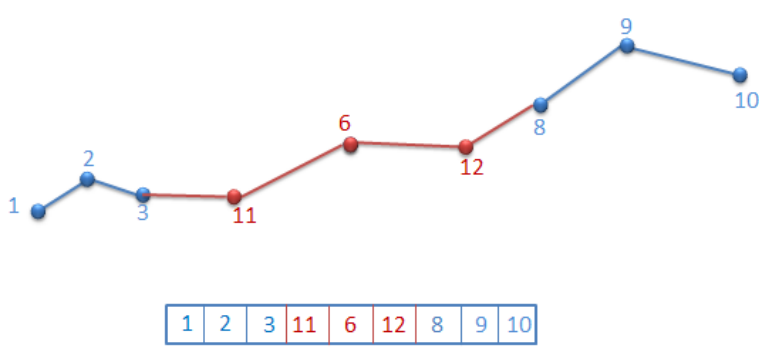

Figure 5. An instance neighbourhood route for the route illustrated in Figure 4.

\section{EXPERIMENTAL RESULTS}

The proposed model was implemented in a GUI MATLAB programming language. The introduced system provides user a configuration wizard to compare criteria.

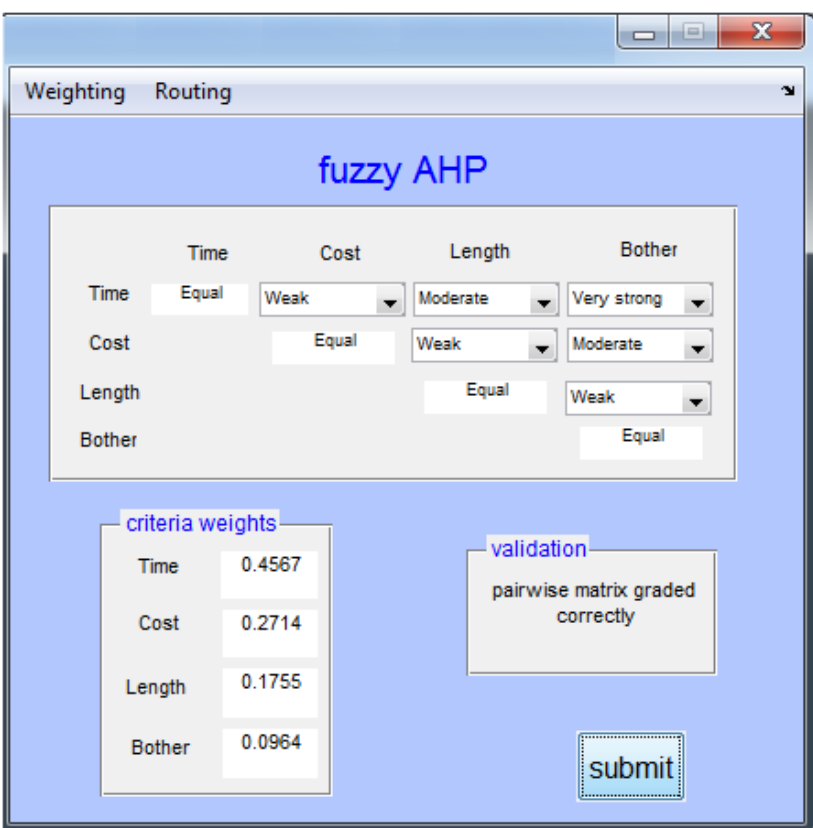

Figure 6. The devoted configuration wizard for the pairwise compression

Afterwards, SA parameters should be tuned. In this paper, for using the proposed SA algorithm efficiently, its parameters, including the population size, the number of neighbours, Boltzmann constant, and the cooling rate have been tuned. At first, to investigate the effects of the population size on the proposed SA algorithm, the Boltzmann constant, the number of neighbours, the cooling rate, and the maximum iteration value were set on 45, 10, 0.5, and 2000 respectively. The population size values were varied from 5 to 80 with increment value 5 . Results showed that the population-size values larger than 20 were not significantly influence on the performance of the proposed SA algorithm. Thus, population size has been tuned on 20 (Figure 7). Similarly, the influence of the other parameters on the proposed SA algorithm were investigated and their value were tuned (Figures 8 to 10).

To verify the proposed model and illustrate its application in real world multi-modal multi-criteria route planning, the transportation network of a central area of Tehran city was used. The proposed model was implemented in an area in centre of Tehran. The considered area is $52.9 \mathrm{~km}^{2}$ that consists 4 BRT lines, 34 sweep bus lines, 4 sweep subway lines, and totally more than $1000 \mathrm{~km}$ of roads (Figure 11).

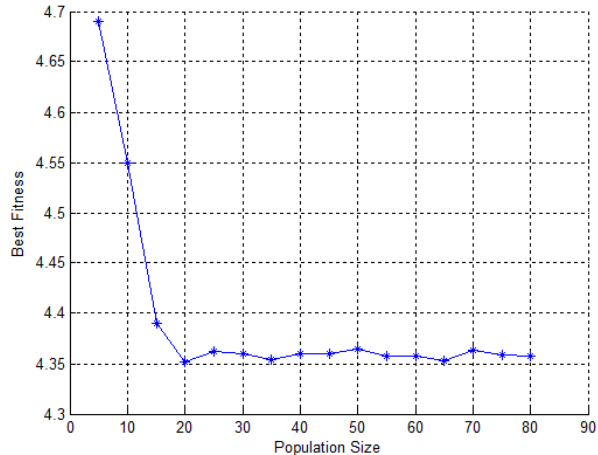

Figure 7. The best fitness value of the achieved routes over 100 independent runs under different population sizes

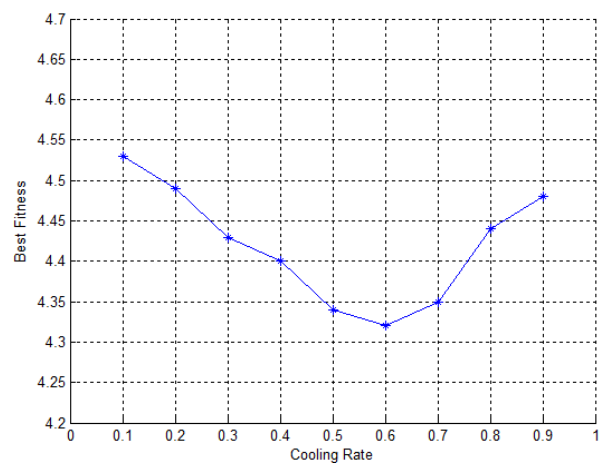

Figure 8 . The best fitness value of the achieved routes over 100 independent runs under different cooling rates.

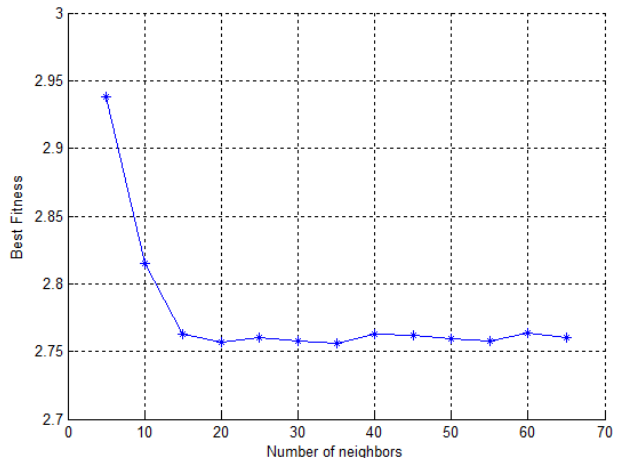

Figure 9. The best fitness value of the achieved routes over 100 independent runs under different neighbour numbers.

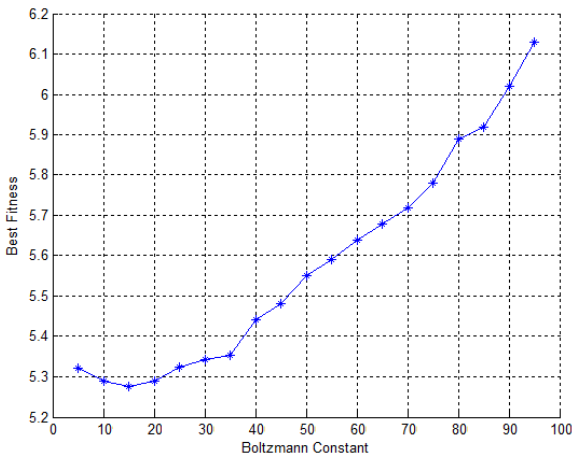

Figure 10. The best fitness value of the achieved routes over 100 independent rus under different Boltzmann Constants. 


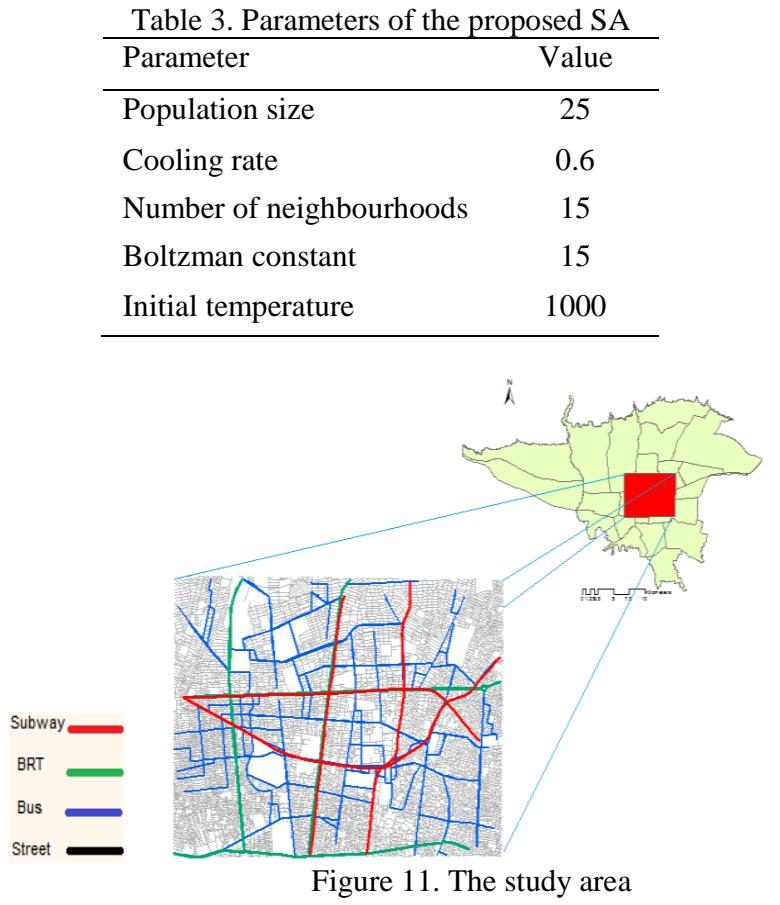

In this research, distances that were lower than 500 meters were considered to be as walking and for those that were bigger than that were considered as other transportation modes. Two examples of this study implementation according to both weights of Figure 6 and determined tuned value of the proposed SA parameters (Table 3) are illustrated in Figures 12 to 15.

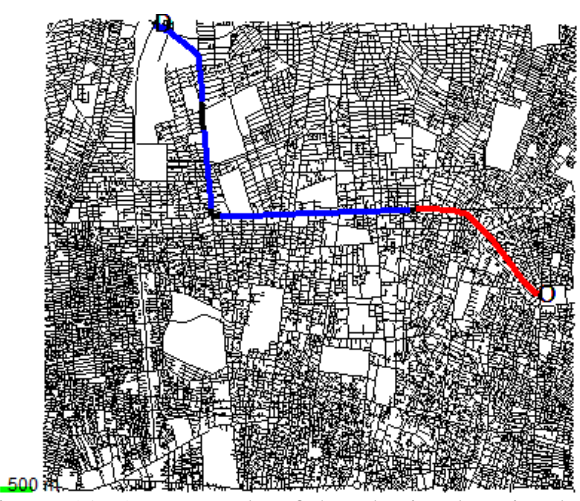

Figure 12. An example of the obtained optimal route according to the weights shown in Figure7 (Length: $8620.2 \mathrm{~m}$, Time $=41.8$ min, Fare $=11500$ Rials)

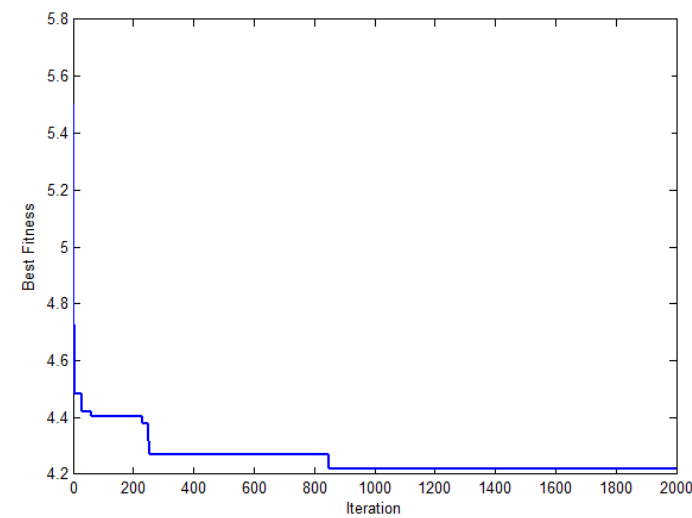

Figure 13. Convergence plot of the optimal route shown in Figure 12.

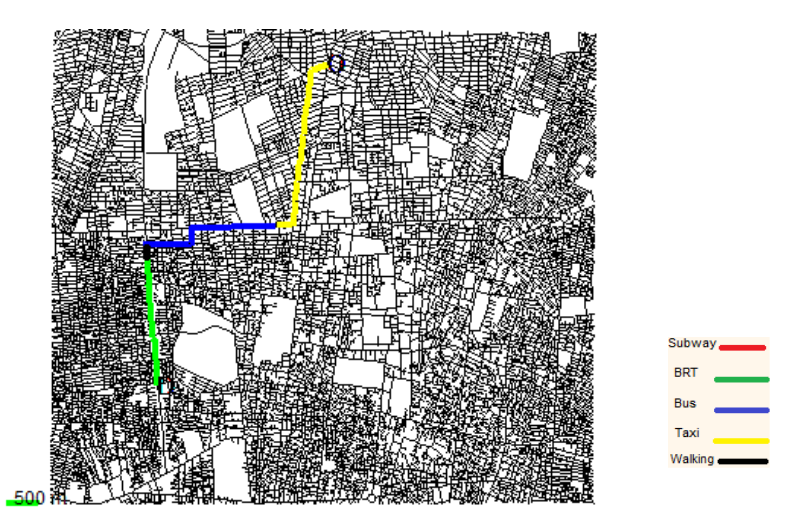

Figure 14. An example of the obtained optimal route according to the weights shown in Figure7 (Length: 6883.7 m, Time $=36.4$ min, Fare $=13500$ Rials)

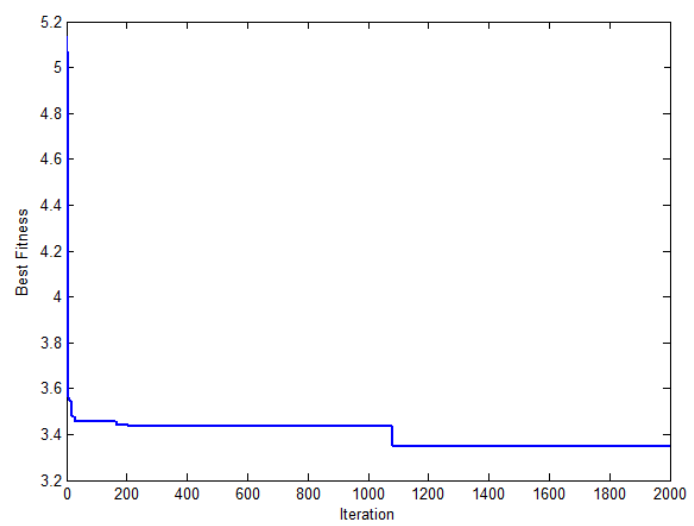

Figure 15. Convergence plot of the optimal route shown in Figure 14.

\section{CONCLUSION}

In this paper, a fuzzy AHP weighting method and a SA algorithm were integrated for finding optimal multimodal route in a real static transportation network. The proposed model was implemented in an area in centre of Tehran in a GUI MATLAB programming language. The results showed a high efficiency and speed of the proposed algorithm that support our analyses. In future studies, performance of the proposed model in a dynamic transportation network that its information could be updated during the implementation of the system will be studied. Also, using multi objective simulate annealing (MOSA) algorithm can be studied in future.

\section{REFERENCES}

Abbaspour, R. A., \& Samadzadegan, F. (2011). Time-dependent personal tour planning and scheduling in metropolises. Expert Systems with Applications, 38(10), 12439-12452.

Abdelghany, K., \& Mahmassani, H. (2001). Dynamic trip assignment-simulation model for intermodal transportation networks. Transportation Research Record: Journal of the Transportation Research Board(1771), 52-60.

Antsfeld, L., \& Walsh, T. (2012). Finding multi-criteria optimal paths in multi-modal public transportation networks using the transit algorithm. Paper presented at the Intelligent Transport Systems World Congress. 
Borole, N., Rout, D., Goel, N., Vedagiri, P., \& Mathew, T. V. (2013). Multimodal Public Transit Trip Planner with Real-time Transit Data. Procedia-Social and Behavioral Sciences, 104, 775-784.

Bouhana, A., Fekih, A., Abed, M., \& Chabchoub, H. (2013). An integrated case-based reasoning approach for personalized itinerary search in multimodal transportation systems. Transportation Research Part C: Emerging Technologies, 31 , $30-50$.

Buckley, J. J. (1985). Fuzzy hierarchical analysis. Fuzzy sets and systems, 17(3), 233-247.

Crainic, T. G., \& Rousseau, J.-M. (1986). Multicommodity, multimode freight transportation: A general modeling and algorithmic framework for the service network design problem. Transportation Research Part B: Methodological, 20(3), 225242.

Davies, C., \& Lingras, P. (2003). Genetic algorithms for rerouting shortest paths in dynamic and stochastic networks. European Journal of Operational Research, 144(1), 27-38.

Delavar, M., Samadzadegan, F., \& Pahlavani, P. (2004). A GISAssisted optimal urban route finding approach based on genetic algorithms. International archives of photogrammetry remote sensing and spatial information sciences, 35(Part 2), 305-308.

Deng, H. (1999). Multicriteria analysis with fuzzy pairwise comparison. International Journal of Approximate Reasoning, 21(3), 215-231.

Huang, B., Cheu, R. L., \& Liew, Y. S. (2004). GIS and genetic algorithms for HAZMAT route planning with security considerations. International Journal of Geographical Information Science, 18(8), 769-787.

Kirkpatrick, S., Gelatt, C. D., \& Vecchi, M. P. (1983). Optimization by simulated annealing. science, 220(4598), 671680.

Leung, L., \& Cao, D. (2000). On consistency and ranking of alternatives in fuzzy AHP. European Journal of Operational Research, 124(1), 102-113.

Liu, L., Mu, H., Luo, H., \& Li, X. (2012). A simulated annealing for multi-criteria network path problems. Computers \& Operations Research, 39(12), 3119-3135.

Liu, L., Yang, J., Mu, H., Li, X., \& Wu, F. (2014). Exact algorithms for multi-criteria multi-modal shortest path with transfer delaying and arriving time-window in urban transit network. Applied Mathematical Modelling, 38(9), 2613-2629.

Malczewski, J. (1999). GIS and multicriteria decision analysis: John Wiley \& Sons.

Nadi, S., \& Delavar, M. R. (2011). Multi-criteria, personalized route planning using quantifier-guided ordered weighted averaging operators. International Journal of Applied Earth Observation and Geoinformation, 13(3), 322-335.

Nguyen, S., Morello, E., \& Pallottino, S. (1988). Discrete time dynamic estimation model for passenger origin/destination matrices on transit networks. Transportation Research Part B: Methodological, 22(4), 251-260.
Pahlavani, P., \& Delavar, M. R. (2014). Multi-criteria route planning based on a driver's preferences in multi-criteria route selection. Transportation Research Part C: Emerging Technologies, 40, 14-35.

Pahlavani, P., Delavar, M. R., \& Frank, A. U. (2012). Using a modified invasive weed optimization algorithm for a personalized urban multi-criteria path optimization problem. International Journal of Applied Earth Observation and Geoinformation, 18, 313-328.

Pahlavani, P., Samadzadegan, F., \& Delavar, M. R. (2006). A GIS-based approach for urban multi-criteria quasi optimized route guidance by considering unspecified site satisfaction Geographic Information Science (pp. 287-303): Springer.

Qu, L., \& Chen, Y. (2008). A hybrid MCDM method for route selection of multimodal transportation network Advances in Neural Networks-ISNN 2008 (pp. 374-383): Springer.

Saaty, T. L. (1980). The analytic hierarchy process: planning, priority setting, resources allocation. New York: McGraw.

Yang, T., Peters, B. A., \& Tu, M. (2005). Layout design for flexible manufacturing systems considering single-loop directional flow patterns. European Journal of Operational Research, 164(2), 440-455.

Yu, H., \& Lu, F. (2012). A multi-modal route planning approach with an improved genetic algorithm. Advances in Geo-Spatial Information Science, 193. 